\title{
CONTRATACIÓN ON-LINE
}

\section{ON-LINE CONTRACTING}

\author{
PABLO MEDINA MAGALLANES \\ MIGUEL ÁNGEL DE LA FUENTE** \\ Fecha de recepción: 14 de octubre 2019 \\ Fecha de aceptación: 11 de marzo de 2020 \\ Disponible en línea: 30 de diciembre 2020
}

Para citar este artículo/To cite this article

\begin{abstract}
Medina Magallanes, Pablo \& De la Fuente, Miguel Ángel. Contratación on-line, 53 Rev. Ibero-Latinoam.Seguros, 117-136 (2020). https://doi.org/10.11144/Javeriana.ris53.coli doi:10.11144/Javeriana.ris53.coli
\end{abstract}

\footnotetext{
* Medina Magallanes, Pablo. Licenciado en Derecho por la Universidad Panamericana, especialista en Derecho Constitucional y Amparo por la Universidad Panamericana y Especialista en Derecho de Seguros por la Universidad de Salamanca. Socio director de Medina Abogados desde 1990. Contacto: pmedina@ medinaabogados.com.mx

** De la Fuente Estrada, Miguel Ángel. Licenciado en Derecho por la Universidad Anáhuac México, con Maestría en Finanzas por la Universidad Anáhuac México y Derecho Financiero Internacional por King's College London, University of London. Asociado principal de la práctica de Derecho Mercantil de Garrigues México, S.C. Contacto: mdelafuente@dacbeachcroft.com
} 


\title{
RESUMEN
}

Frente al surgimiento de las nuevas tecnologías, la regulación tradicional enfrenta desafíos diversos para poder adaptarse a las cambiantes circunstancias económicas y sociales que todo esto conlleva. El objeto del presente artículo es hablar de manera específica sobre la aplicación de las nuevas tecnologías al contrato de seguro, estableciendo los retos a los que se enfrenta el actual panorama regulatorio y los principales elementos para regular las nuevas tecnologías emergentes. Se busca explicar, desde una perspectiva legal, el funcionamiento de la contratación electrónica de seguros en México y establecer algunas consideraciones de lo que podría ser tomado en cuenta por la regulación para resolver algunas de las problemáticas esenciales que enfrenta estas nuevas formas de canales de comercialización de productos de seguros.

Palabras clave: Insurtech, nuevas tecnologías, operaciones electrónicas, contratación electrónica, intermediación, regulación tradicional.

\begin{abstract}
In the midst of the development of new technologies, traditional regulation is facing various challenges to adapt to the changing economic and social environment. The purpose of this article is to discuss the application of new technologies to the insurance contract, outlining those challenges faced by the current regulatory landscape and the main elements for regulating new emerging technologies. The aim is to explain from a legal perspective, how electronic insurance contracting works in Mexico, and to establish some considerations as to the way regulation may approach some of the essential problematics faced by this new insurance products commercialization channels.
\end{abstract}

Keywords: Insurtech, new technologies, electronic transactions, electronic insurance contracting, intermediation, traditional regulation.

\section{SUMARIO}

1. Introducción - 1.1. Panorama regulatorio mexicano - 1.2. Desafíos de la regulación tradicional - 1-3. Principios para la regulación de las tecnologías emergentes -2 . Tipos de operaciones electrónicas -2.1 Operaciones electrónicas móviles -2.2 Operaciones electrónicas de audio respuesta -2.3 operaciones host to host -2.4 operaciones electrónicas por internet -2.5 Operaciones telefónicas voz a voz -3 . Intermediación de seguros - 3.1 Plataformas digitales - 3.2 Comparadores, agregadores y robots asesores (roboadvisors) - 3.3 Intermediación Peer-to-peer - 4. Contratos de seguro electrónico - $4.1 \mathrm{On}$ demand - 4.2 Peer-to-peer- 4.3 Usage based - 5. Principios de contratación electrónica -6 . Principales disposiciones contractuales para la contratación electrónica -7 . Factores de autenticación -8 . Entrega de documentación contractual al asegurado -9 . Mecanismos de protección de asegurados -9.1 Criterios sobre sanas prácticas, transparencia y publicidad - 9.2 Protección de datos y privacidad - 9.3 Ventas atadas - 9.4 Alfabetización financiera - 10 . Conclusiones -11 . Bibliografía. 


\section{INTRODUCCIÓN}

\subsection{Panorama regulatorio mexicano}

La contratación on-line de seguros en México es regulada por:

Ley de Instituciones de Seguros y Fianzas (“LISF”).

Art. 214.- La celebración de las operaciones y la prestación de servicios de las Instituciones, se podrán pactar mediante el uso de equipos, medios electrónicos, ópticos o de cualquier otra tecnología, sistemas automatizados de procesamiento de datos y redes de telecomunicaciones, ya sean privados o públicos, estableciendo en los contratos respectivos las bases para determinar lo siguiente:

I. Las operaciones y servicios cuya prestación se pacte;

II. Los medios de identificación del usuario, así como las responsabilidades correspondientes a su uso, tanto para las Instituciones como para los usuarios;

III. Los medios por los que se hagan constar la creación, transmisión, modificaciones o extinción de derechos y obligaciones inherentes a las operaciones y servicios de que se trate, incluyendo los métodos de autenticación tales como contraseñas o claves de acceso, y

IV. Los mecanismos de confirmación de la realización de las operaciones celebradas a través de cualquier medio electrónico.

El uso de los medios de identificación que se establezcan conforme a lo previsto por este artículo, en sustitución de la firma autógrafa, producirá los mismos efectos que las leyes otorgan a los documentos correspondientes y, en consecuencia, tendrán el mismo valor probatorio.

La instalación y el uso de los equipos y medios señalados en el primer párrafo de este artículo se sujetarán a las disposiciones de carácter general que, en su caso, emita la Comisión.

Circular Única de Seguros y Fianzas (“CUSF”).

Ley Modelo Sobre Comercio Electrónico aprobada por la CNUDMI/ UNCITRAL (Comisión de las Naciones Unidas para la Unificación del Derecho Mercantil Internacional).

Disposiciones de Carácter General en Materia de Sanas Prácticas, Transparencia y Publicidad Aplicables a las Instituciones de Seguros emitidas por la Comisión Nacional para la Protección y Defensa de los Usuarios de Servicios Financieros (“CONDUSEF”).

\subsection{Desafios de la regulación tradicional}

Una de las principales problemáticas que enfrenta en la actualidad la regulación tradicional frente al surgimiento de diferentes tecnologías, como puede ser Insurtech, es 
el problema del ritmo, puesto que las estructuras regulatorias existentes a menudo son lentas para adaptarse a las cambiantes circunstancias económicas y sociales y por lo tanto el enfoque regulatorio actual no es el más óptimo para respaldar el rápido ritmo de desarrollo que llevan estas tecnologías. Si bien el ciclo de las políticas lleva de cinco a veinte años, un emprendimiento de un billón de dólares puede convertirse en una empresa con alcance global en cuestión de meses, tales son los casos de empresas como Uber y Airbnb.

Otro de los problemas que enfrenta la regulación tradicional son las formas disruptivas de cambio tecnológico que cruzan los límites tradicionales de la industria, ya que es difícil mantener la coherencia en las reglas y regulaciones de una economía colaborativa en que, por lo general, la responsabilidad se divide entre proveedores, facilitadores y clientes.

También han surgido dudas sobre la regulación que debe aplicarse al uso de teléfonos inteligentes, dispositivos conectados a la red y sensores, ya que existe una disyuntiva sobre si el poseedor de estos datos es el usuario o el proveedor de servicios que lo almacena y de ser el último caso, bajo qué reglas lo hace y en qué medida puede compartirlos con terceros. Si bien, ya hay algunos países que se han dedicado a la labor de regular este tema, por lo general tienen leyes contradictorias tanto a nivel interno como a nivel internacional.

Ante la pregunta sobre si las nuevas tecnologías deberían ser reguladas a nivel interno o bien crear una regulación uniforme a nivel internacional surgió un problema denominado el problema de la "caja negra", el cual plantea la complejidad que tienen los algoritmos actuales y por tanto la importancia por comprenderlos, pero al estar tan estrechamente ligados con las organizaciones que los desarrollan hacen que se vuelva imposible para los legisladores ver qué hay dentro de un algoritmo y por tanto regularlo. Se ha planteado que el funcionamiento de los algoritmos sea abierto al público, pero esto entraría en conflicto con diversos acuerdos de confidencialidad que mantienen las diferentes empresas. Dentro del mismo tema de los algoritmos, diferentes estudios han revelado que muchos de éstos tienen sesgos inherentes que podrían considerarse discriminatorios, por ejemplo, hay anuncios que únicamente les aparecen a personas cuyos nombres podrían indicar que se trata de personas de color, mientras que a personas cuyos nombres indicaran lo contrario jamás les aparecerían estos anuncios.

En el caso específico de Insurtech, es decir las nuevas tecnologías aplicadas a la industria de los seguros, los más recientes modelos de comercialización podrían infringir determinadas normas establecidas para la mediación, como es el caso de la figura de la intermediación Peer-to-peer, en que la figura de la entidad intermediaria puede llegar a superar los límites de la intermediación en sentido estricto, ya que no solo gestiona colectivamente la contratación de las pólizas con vistas a obtener de la aseguradora mejores condiciones frente a las que se obtendrían si se negociasen las contrataciones de forma individual, si no que puede intervenir indirectamente en la delimitación y en la cobertura del riesgo. 
Para poder llegar a una mejor regulación debemos seguir cuatro diferentes etapas en las que en primer lugar debemos preguntarnos cuál es el estado actual de la regulación para darnos cuenta de todas aquellas legislaciones que pudieran estar bloqueando una apertura a la innovación, que estén obsoletas o bien estén duplicadas y puedan llegar a ser contradictorias. En segundo lugar, es importante plantearse cuál es el momento adecuado para regular para evitar que se haga demasiado rápido o demasiado lento y además poder transitar a un modelo regulador más ágil. La tercera fase debe responder a la pregunta de cuál es el enfoque correcto para la regulación, la cual podrá tener varias respuestas dependiendo de a qué decidan darle mayor importancia los legisladores: protección a los ciudadanos, promover la competencia o internalizar las externalidades. La cuarta pregunta a plantearse debe responder a qué ha cambiado desde que se inició con la regulación, la cual probablemente sea contestada hasta la próxima década.

\subsection{Principios para la regulación de tecnologias emergentes}

Cinco principios para guiar el futuro de la regulación:

\subsubsection{Regulación adaptativa. - Cambio del "regula y olvida" a la aproximación sensible e iterativa}

Este principio busca enfoques adaptativos a la regulación, basados en la prueba y error, en que haya un diseño conjunto de la regulación y de los estándares y además haya ciclos de retroalimentación más rápidos para poder comparar las políticas con los estándares y proporcionar los resultados en una revisión de la regulación.

Otra de las propuestas que se tiene para cumplir con este principio es la aplicación de mecanismos soft law, como lo pueden ser la orientación informal, el impulso para auto regulación de la industria, guía de mejores prácticas, códigos de conducta y acreditación de terceros. Si bien estos mecanismos no son legalmente vinculantes, permiten a los reguladores adaptarse rápidamente a los cambios y abordar las problemáticas que surjan sin bloquear la innovación, a diferencia de los mecanismos hard law. Una forma eficiente de aplicar estos mecanismos sería hacerlo de manera interna en la propia industria definiendo el alcance de los problemas a abordar y solicitando a la industria que desarrolle sus propios estándares y códigos de conducta, lo cual ya ha sucedido en países como Finlandia en cuanto a la regulación del transporte.

\subsubsection{Aceleradores reguladores. Aproximaciones basadas en prototipos y pruebas por medio de la creación de sandbox's $y$ aceleradores}

Este principio busca que se asocien empresas privadas y empresarios para experimentar nuevas tecnologías en entornos que propicien la innovación. Este es el caso 
de los sandbox, los cuales son entornos controlados que permiten a los innovadores probar productos, servicios o nuevos modelos comerciales sin tener que seguir todas las normas estándar, como lo es el caso de las cripto monedas en Canadá o los sistemas aéreos en Estados Unidos, y además ayuda a los reguladores a entender mejor las nuevas tecnologías y colaborar con la industria para desarrollar apropiadamente las reglas y regulaciones que las rijan apropiadamente.

\subsubsection{Regulación basada en resultados. Enfoque en resultados y rendimiento más que en la forma}

El hecho de que los gobiernos cambien el enfoque de las regulaciones de los insumos a los resultados puede crear eficiencias operacionales para los reguladores y una mayor libertad para los innovadores, ya que lo que busca es especificar los resultados que deben generarse en lugar de la manera en que deben generarse y además proporcionar el margen necesario para experimentar.

\subsubsection{Regulación de riesgo ponderado. Salto de la regulación "un caso aplica a todos" hacia la aproximación segmentada y basada en información}

Una forma de acelerar la aprobación de modelos de negocios basados en tecnologías emergentes es inspirarse en los sistemas de comprobación previa para viajes aéreos en que se podría usar un enfoque similar para ayudar a agilizar las aprobaciones de nuevos modelos de negocio, permitiendo que ciertas empresas pasen por un proceso de aprobación optimizado y predecible dependiendo de que proporcionen acceso a la información clave. Este sistema basado en los datos y en el riesgo no debe limitarse a la aprobación previa, sino que puede extenderse a un enfoque dinámico y regulatorio basado en flujos de datos en tiempo real entre las empresas y sus reguladores. Basándose en el análisis de los resultados al compararse con la regulación y los resultados esperados se podría decidir si una empresa cumple y por tanto será clasificada como segura y de lo contrario emitir una lista de requisitos para alcanzar dicha categoría o las sanciones que se deberán imponer a violaciones mayores.

\subsubsection{Regulación colaborativa. Alineación nacional e internacional al engranar un grupo de jugadores más amplio a lo largo del ecosistema}

Debido al crecimiento de la economía digital, los nuevos modelos comerciales, tecnologías, productos y servicios, los reguladores a nivel mundial podrían beneficiarse de la co-regulación, la autorregulación y la coordinación internacional, ya que por medio de acuerdos se podría llegar a marcos estándares y directrices internacionales. 


\section{TIPOS DE OPERACIONES ELECTRÓNICAS}

\subsection{Operaciones electrónicas móviles}

Son las operaciones electrónicas en las cuales el Dispositivo de Acceso consiste en un Teléfono Móvil del Usuario, cuyo número de línea se encuentre asociado al servicio.

\subsection{Operaciones electrónicas de audio respuesta}

Son las las Operaciones Electrónicas mediante las cuales la Institución o Sociedad Mutualista recibe instrucciones del Usuario a través de un sistema telefónico, e interactúa con el propio Usuario mediante grabaciones de voz y tonos o mecanismos de reconocimiento de voz, incluyendo los sistemas de respuesta interactiva de voz (IVR).

\subsection{Operaciones host to host}

Operaciones Electrónicas mediante las cuales se establece una conexión directa entre los equipos de cómputo del Usuario previamente autorizados por la Institución o Sociedad Mutualista y los equipos de cómputo de la propia Institución o Sociedad Mutualista, a través de los cuales estos últimos procesan la información para la realización de servicios y operaciones. Este tipo de servicios incluirán a los proporcionados a través de las aplicaciones conocidas como "Cliente-Servidor".

\subsection{Operaciones electrónicas por internet}

Operaciones Electrónicas efectuadas a través de la red electrónica mundial denominada Internet, en el sitio que corresponda a uno o más dominios de la Institución o Sociedad Mutualista, incluyendo el acceso mediante el protocolo WAP o alguno equivalente.

\subsection{Operaciones telefónicas voz a voz}

Operaciones Electrónicas mediante las cuales un Usuario instruye vía telefónica a través de un empleado o representante de la Institución o Sociedad Mutualista autorizado por ésta, con funciones específicas, el cual podrá operar en un centro de atención telefónica, a realizar operaciones a nombre del propio Usuario.

\section{INTERMEDIACIÓN DE SEGUROS}

\subsection{Plataformas digitales}

Se refiere al uso de plataformas por medio de internet o smartphones (teléfonos inteligentes), las cuales han surgido como iniciativas que buscan mejorar la experiencia 
y servicio proporcionado al cliente, así como llegar a nuevos mercados. Por medio de estas tecnologías se busca cubrir con las demandas de los consumidores por medio de la inmediata provisión de bienes y servicios. Estas tecnologías, además, ayudan a las aseguradoras a tomar el control del proceso de reclamos que presenten los clientes de una manera integral y a incorporar seguros Peer-to-peer, en que grupos con intereses similares puedan negociar y ponerse de acuerdo para la cobertura de sus pólizas de manera conjunta.

\subsection{Comparadores, agregadores $y$ robots asesores (roboadvisors)}

Se trata de servicios en línea que proporcionan comparación de productos y asesoría de manera automática, por medio de algoritmos y sin intervención humana. Estos servicios pueden proporcionar respuestas más o menos individualizadas conforme a la información proporcionada por el usuario y es por esto que además de ofrecer productos, son útiles para resolver consultas o definir la cobertura adecuada para el usuario.

\subsection{Intermediación peer-to-peer}

Este tipo de seguro permite a los usuarios reunir su dinero, organizar y administrar su seguro de manera personal. La intermediación Peer- to- Peer, siendo su traducción al español "red de pares", se refiere a los servicios en que hay mayor participación de los propios usuarios en la prestación de éstos puesto que utilizan las redes sociales para formar grupos de personas que contratan un mismo tipo de seguro y de esta manera cuentan con un perfil de riesgo similar.

Una de las secuencias de comercialización de seguros más comunes de intermediación Peer-to-peer es aquella en que la entidad intermediaria se dedica a agrupar tomadores con el mismo tipo de seguro y destina una parte de su prima a crear una reserva común contra la cual se cargarán los pagos de las indemnizaciones de menor importe y es así como aquellas reclamaciones que superen el importe de la reserva serán cubiertas por parte de la aseguradora, mientras que las que puedan pagarse con cargo a la referida reserva no producirán ningún impacto en la póliza contratada con la aseguradora.

\section{CONTRATOS DE SEGURO ELECTRÓNICO}

\subsection{On demand}

Se trata de un nuevo modelo de negocio que se especializa en cubrir únicamente aquellos riesgos abordados en determinado momento. Un ejemplo de este tipo de contrato de seguro se puede ver con la aseguradora Cuvva, la cual permite el seguro de un coche desde el momento en que empieza a ser conducido hasta el momento en que llega a su destino. 


\subsection{Peer-to-peer}

Es un modelo de negocio que permite a los asegurados acumular su dinero, auto organizar y auto administrar su propio seguro, ya que la idea nuclear de este tipo de seguros es que un grupo de personas con la misma mentalidad y los mismos intereses unan sus pólizas de seguros para así tener mayor control, confianza y transparencia y al mismo tiempo reducir costos.

\subsection{Usage based}

Se trata de un nuevo modelo de negocio introducido por auto aseguradoras, el cual alinea de manera más cercana conductas al volante con tasas de primas para seguro de automóviles. El kilometraje y las conductas al volante son rastreadas por medio de Telematics, el cual monitorea de manera directa a la persona mientras conduce. La institución de seguros analiza la información recopilada y establece tasas de primas conforme a ello.

\section{PRINCIPIOS DE CONTRATACIÓN ELECTRÓNICA}

Equivalencia funcional de los actos empresariales electrónicos. - De este principio se derivan las disposiciones fundamentales que regulan esta nueva actividad mercantil. Conforme a Illescas Ortiz, este principio se define de la siguiente manera:

"La función jurídica que en toda su extensión cumple la instrumentación escrita y autógrafa -o eventualmente su expresión oral- respecto de cualquier acto jurídico, la cumple igualmente su instrumentación electrónica a través de un mensaje de datos, con independencia del contenido, dimensión, alcance y finalidad del acto así instrumentado. La equivalencia funcional, en suma, implica aplicar a los mensajes de datos electrónicos una pauta de no discriminación respecto de las declaraciones de voluntad o ciencia manual, o gestualmente efectuadas por el mismo sujeto".

Conforme a esta definición podemos entender que las funciones que cumplen los documentos en papel igualmente las cumplen por medios electrónicos, e incluso pueden llegar a ser más seguras que los medios tradicionales.

Inalterabilidad del derecho preexistente de obligaciones y contratos privados. Se pretende que las reglas que regulan el comercio electrónico no impliquen una modificación sustancial del derecho existente de las obligaciones y de los contratos nacionales e internacionales en cuanto a la articulación jurídica electrónica como instrumento de transacciones comerciales, ya que los medios electrónicos únicamente suponen un nuevo soporte y medio de transmisión, pero no un nuevo derecho regulador de éstos y su sentido jurídico.

Neutralidad tecnológica. La doctrina ha definido este principio como la aptitud que debe imperar en las nuevas normas del comercio electrónico para abarcar con sus reglas no sólo las tecnologías existentes en el momento en que se formulan, sino también las tecnologías futuras, sin necesidad de verse sometidas a modificación, ello, 
obviamente, en un horizonte cronológico razonable. Se busca que estas normas sean aplicables al comercio electrónico y no a una concreta tecnología, por lo que deben tener cierto grado de capacidad e idoneidad para abarcar e incorporar sus reglas o principios no sólo a la tecnología existente al momento en que fueron formuladas, sino también para aquellas tecnologías futuras.

Principio de la buena fe. Este principio es un principio general del derecho que implica que, en la celebración de los negocios, las partes tiene la obligación moral de cumplir a cabalidad con lo pactado. En cuanto al comercio electrónico, Illescas Ortiz establece que debe verse bajo tres diferentes ópticas: en primer lugar, la buena fe debe verse como una manifestación en concreto del principio de inalterabilidad del derecho preexistente de las obligaciones privadas en el ámbito de la contratación electrónica; en segundo lugar, debe verse como un postulado centrado en el concepto de la confianza, pero que debe afirmarse con más fuerza en la contratación electrónica que en una contratación verbal o manuscrita, y por último, debe considerarse como uno de los fundamentos del régimen jurídico aplicable al intercambio internacional y comercial de bienes y servicio.

Autonomía de la voluntad mantenida en el nuevo contexto del comercio electrónico. Debido a que es la libertad de las partes para llegar a pactos o acuerdos, mientras no haya prohibición, la norma que rige los contratos privados, no es necesaria ninguna norma que habilite para la utilización de medios electrónicos en la contratación, ya que la regulación busca facilitar el comercio electrónico, pero no imponerlo a toda costa, respetando así la autonomía de la voluntad.

\section{PRINCIPALES DISPOSICIONES CONTRACTUALES PARA LA CONTRATACIÓN ELECTRÓNICA}

En el año 1996, la Comisión de las Naciones Unidas para el Derecho Mercantil Internacional (CNUDMI), dependiente de la ONU, aprobó la Ley Modelo sobre el Comercio Electrónico. La misma contempló un conjunto de reglas que pueden ser tomadas como base para la subsecuente creación y/o reformas de leyes en materia de comercio electrónico, con el fin de evitar obstáculos jurídicos para el desarrollo de contratación mediante medios electrónicos.

Para efectos de lo anterior, en la misma norma se determinó en su artículo 11, la formación y validez de los contratos mediante los medios electrónicos:

Art. 11. - Formación y validez de los contratos

1) En la formación de un contrato, de no convenir las partes otra cosa, la oferta y su aceptación podrán ser expresadas por medio de un mensaje de datos. No se negará validez o fuerza obligatoria a un contrato por la sola razón de haberse utilizado en su formación un mensaje de datos.

En consecuencia de lo anterior, no se determinó algún elemento de validez distinto al que la Teoría General de las Obligaciones señala como elementos para la celebración de un contrato, es decir el objeto y el consentimiento. 
Para el año 2000 (CCF, CFPC y CCO) y 2003 (CCO), México adoptó los principios y términos que de la Ley Modelo de la sobre el Comercio Electrónico de la CNUDMI, reformando leyes como el Código Civil Federal, Código Federal de Procedimientos Civiles y El Código de Comercio, sin que la Ley Sobre el Contrato de Seguro contuviese modificación alguna al respecto.

a) Para efectos de lo anterior, en el Código Civil Federal (año 2000), se reformó dentro del libro cuarto “de las obligaciones", los arts. 1803, 1805 1811, así como la adición del numeral 1834 bis,

Art. 1803.- El consentimiento puede ser expreso o tácito, para ello se estará a lo siguiente:

I.- Será expreso cuando la voluntad se manifiesta verbalmente, por escrito, por medios electrónicos, ópticos o por cualquier otra tecnología, o por signos inequívocos, y

II.- El tácito resultará de hechos o de actos que lo presupongan o que autoricen a presumirlo, excepto en los casos en que por ley o por convenio la voluntad deba manifestarse expresamente.

Art. 1805.- Cuando la oferta se haga a una persona presente, sin fijación de plazo para aceptarla, el autor de la oferta queda desligado si la aceptación no se hace inmediatamente. La misma regla se aplicará a la oferta hecha por teléfono o a través de cualquier otro medio electrónico, óptico o de cualquier otra tecnología que permita la expresión de la oferta y la aceptación de ésta en forma inmediata.

b) Como bien es conocido, el Contrato de Seguro es considerado un acto de comercio en términos de la fracción XVI del art. 75 del Código de Comercio, legislación que adoptó los lineamientos de la Ley Modelo sobre el Comercio Electrónico de la CNUDMI (año 2003), por lo que en materia de seguros es aplicable el Título Segundo del libro segundo de dicha legislación, denominado "De Comercio Electrónico" para lo cual se establece:

Art. 89.- Las disposiciones de este Título regirán en toda la República Mexicana en asuntos del orden comercial, sin perjuicio de lo dispuesto en los tratados internacionales de los que México sea parte.

En los actos de comercio y en la formación de los mismos podrán emplearse los medios electrónicos, ópticos o cualquier otra tecnología.

Art. 89 bis. - No se negarán efectos jurídicos, validez o fuerza obligatoria a cualquier tipo de información por la sola razón de que esté contenida en un Mensaje de Datos. Por tanto, dichos mensajes podrán ser utilizados como medio probatorio en cualquier diligencia ante autoridad legalmente reconocida, y surtirán los mismos efectos jurídicos que la documentación impresa, siempre y cuando los mensajes de datos se ajusten a las disposiciones de este Código y a los lineamientos normativos correspondientes. 
c) No obstante lo anterior, la Ley de Instituciones de Seguros y Fianzas, del año 2013, siguió los principios adoptados por la Ley Modelo sobre el Comercio Electrónico de la CNUDMI, tal como se desprende del art. 214 citado con anterioridad, por el cual se otorga la facultad a las Instituciones constituidas en arreglo a dicho régimen, para otorgar los servicios financieros de seguros y fianzas mediante medios electrónicos:

Art. 214.- La celebración de las operaciones y la prestación de servicios de las Instituciones, se podrán pactar mediante el uso de equipos, medios electrónicos, ópticos o de cualquier otra tecnología, sistemas automatizados de procesamiento de datos y redes de telecomunicaciones, ya sean privados o públicos, estableciendo en los contratos respectivos las bases para determinar lo siguiente:

I. Las operaciones y servicios cuya prestación se pacte;

II. Los medios de identificación del usuario, así como las responsabilidades correspondientes a su uso, tanto para las Instituciones como para los usuarios;

III. Los medios por los que se hagan constar la creación, transmisión, modificaciones o extinción de derechos y obligaciones inherentes a las operaciones y servicios de que se trate, incluyendo los métodos de autenticación tales como contraseñas o claves de acceso, y

IV. Los mecanismos de confirmación de la realización de las operaciones celebradas a través de cualquier medio electrónico.

El uso de los medios de identificación que se establezcan conforme a lo previsto por este artículo, en sustitución de la firma autógrafa, producirá los mismos efectos que las leyes otorgan a los documentos correspondientes y, en consecuencia, tendrán el mismo valor probatorio.

La instalación y el uso de los equipos y medios señalados en el primer párrafo de este artículo se sujetarán a las disposiciones de carácter general que, en su caso, emita la Comisión.

d) Reglamentario al numeral transcrito, se expidieron los capítulos 4.10. y 4.11 de la Circular única de Seguros y Fianzas, los cuales a grandes rasgos establecieron la forma y requisitos de la contratación de los contratos de seguro y de fianza, mediante medios electrónicos, como lo son las siguientes reglas:

Consentimiento expreso mediante firma autógrafa, firma electrónica avanzada, operaciones electrónicas móviles, operaciones electrónicas vía internet, operaciones electrónicas de audio respuesta y/o operaciones telefónicas de voz a voz.

Así mismo, contempla la creación de un usuario electrónico para los contratantes, así como las medidas de seguridad y/o factores de autenticación al momento de la contratación del seguro, así como operaciones posteriores a ellas, medidas de seguridad que se encuentran divididas en cuatro categorías:

Categoría 1: Información obtenida mediante cuestionarios al usuario por parte de operadores telefónicos, en donde se requieren datos que el usuario conozca. 
Categoría 2: Contraseñas y o Número de Identificación Personal (NIP).

Categoría 3: Información contenida o generada por medios o dispositivos electrónicos y generadores de contraseñas dinámicas de un solo uso.

Categoría 4: Características propias del usuario, tal como lo son huellas dactilares, patrones de iris o retinas, entre otras.

Por último, el capítulo 4.11., establece las bases a las que se deberán de apegar las Instituciones de Seguros para incorporar en las condiciones generales de los productos que ofrezcan al público, un clausulado para la entrega de la documentación contractual:

Póliza de seguro, número de póliza, nombre comercial del producto, así como la identificación para la consulta del clausulado donde consten los derechos y obligaciones (condiciones generales de seguro).

e) Como regulación indirecta, en el mes de marzo de 2018, fue expedida la Ley para Regular las Instituciones de Tecnología Financiera, la cual faculta a las instituciones prestadoras de servicios financieros (tal como lo es el contrato de seguro), otorgar sus servicios mediante medios innovadores (aplicaciones, softwares, bases de datos, etc.):

Art. 1.- La presente Ley es de orden público y observancia general en los Estados Unidos Mexicanos y tiene por objeto regular los servicios financieros que prestan las instituciones de tecnología financiera, así como su organización, operación y funcionamiento y los servicios financieros sujetos a alguna normatividad especial que sean ofrecidos o realizados por medios innovadores.

De toda la legislación transcrita, se desprende que México se encuentra a la vanguardia de la celebración de acuerdos de voluntades mediante medios electrónicos, teniendo una regulación basta y de conformidad a Ley Modelo de la sobre el Comercio Electrónico de la CNUDMI.

\section{FACTORES DE AUTENTICACIÓN}

La CUSF regula en su primer artículo, en su apartado de definiciones, lo que es un Factor de Autenticación, al establecer que se trata de mecanismos tangibles o intangibles, basados en las características físicas del usuario, en dispositivos o información que únicamente el usuario conozca. Este mismo artículo hace un listado en el que incluye aquellos mecanismos que pueden considerarse factores de autenticación y son los siguientes: información que el usuario conozca y que la institución aseguradora valide por medio de cuestionarios practicados por operadores de centros de atención telefónica; información que solamente el usuario conozca, como pueden ser contraseñas o Números de Identificación Personal (NIP); información contenida o generada en medios o dispositivos respecto de los cuales el usuario tenga posesión, como pueden ser dispositivos o mecanismos generadores de contraseñas dinámicas de un solo uso y tarjetas con circuito integrado, que tengan propiedades que impidan la duplicación de dichos medios o dispositivos de la información que estos contengan o generen; e 
información del usuario derivada de sus propias características físicas, como puede ser la huella dactilar.

Esta misma circular, más adelante amplía el contenido sobre Factores de Autenticación, al establecer que las instituciones de seguros podrán permitir a los usuarios la contratación de servicios y operaciones adicionales o modificar los anteriormente contratados por medio de operaciones electrónicas mientras requieran un segundo Factor de Autentificación adicional al utilizado para el inicio de una sesión, el cual deberá ser único para cada usuario y permitirá a la institución de seguros identificar todas las operaciones electrónicas realizadas por el usuario. Se solicita a los usuarios el uso de estos mismos factores para realizar otras operaciones como pueden ser recibir estados de cuenta por medio de correo electrónico, la modificación de del medio de notificación de los servicios de operaciones electrónicas ofrecidos en terminales de punto de venta mediante un centro de atención telefónica y desbloquear el uso de factores de autenticación que previamente hayan sido bloqueados, entre otros.

\section{ENTREGA DE DOCUMENTACIÓN CONTRACTUAL AL ASEGURADO}

El art. 20 de la Ley Sobre el Contrato de Seguro, determina que la aseguradora se encuentra obligada a entregar la documentación contractual correspondiente al producto del seguro contratado:

Art. 20.- La empresa aseguradora estará obligada a entregar al contratante del seguro, una póliza en la que consten los derechos y obligaciones de las partes. La póliza deberá contener:

VIII.- Las demás cláusulas que deban figurar en la póliza, de acuerdo con las disposiciones legales, así como las convenidas lícitamente por los contratantes.

En virtud de ello, la ya multicitada Circular Única de Seguros y Fianzas, determina en su capítulo 4.11. "De la comercialización de productos de seguros de adhesión a través de medios electrónicos", el cual determina lo siguiente:

Para los efectos del art. 214 de la LISF:

4.11.1. El presente Capítulo da a conocer las bases a las que se deberán apegar las Instituciones de Seguros para incorporar en las condiciones generales de los productos que ofrezcan al público, una cláusula relacionada con la entrega de la documentación contractual de los productos de seguros de adhesión que se comercialicen a través de Medios Electrónicos o de un prestador de servicios a los que se refieren los arts. 102, primer párrafo, y 103, fracciones I y II, de la LISF.

4.11.2. Las Instituciones de Seguros, en los contratos de adhesión que celebren bajo la comercialización a través de Medios Electrónicos, o de un prestador de servicios a los que se refieren los arts. 102, primer párrafo, y 103, fracciones I y II, de 
la LISF, cuyo cobro de prima se realice con cargo a una cuenta bancaria o tarjeta de crédito, deberán incluir una cláusula relativa a la entrega de la póliza que deberá contener cuando menos las siguientes bases, sin que puedan incorporarse estipulaciones adicionales que las contradigan o limiten su propósito:

I. Prever que la Institución de Seguros tendrá la obligación de hacer del conocimiento del asegurado la forma en que le proporcionará la póliza, certificado individual o cualquier otro documento que contenga derechos u obligaciones para las partes derivados del contrato celebrado, en un plazo de treinta días naturales contados a partir de la fecha de contratación del seguro. En caso de que el último día para la entrega de la documentación sea inhábil, se entenderá que la misma deberá entregarse el día hábil inmediato siguiente;

II. Señalar el mecanismo de entrega de los documentos a que se refiere la fracción I de esta Disposición, de forma tal que permita verificar a la Comisión que se ha dado cumplimiento a la obligación referida en dicha fracción;

III. La Institución de Seguros, en la cláusula a que se refiere esta Disposición, se obligará a indicar al contratante o asegurado los medios alternos con que contará para obtener la póliza, $\mathrm{y}$

IV. Establecer el mecanismo para cancelar la póliza o solicitar que no se renueve automáticamente la misma.

4.11.3. Al acordar la contratación del seguro en términos de la Disposición 4.11.2, las Instituciones de Seguros deberán proporcionar al contratante o asegurado:

I. El número de póliza o folio de confirmación que corresponda a su solicitud de contratación, mismo que servirá como prueba en caso de alguna aclaración;

II. El nombre comercial del producto de seguro o los datos de identificación del mismo;

III. La dirección de la página electrónica que la Institución de Seguros deberá mantener en Internet, con la finalidad de que el Usuario pueda identificar y consultar el modelo del clausulado en donde consten los derechos y obligaciones adquiridos;

IV. Los datos de contacto para la atención de siniestros o quejas de la Institución de Seguros con la que se contrató el producto;

V. La información y datos de contacto para efectuar la cancelación de la póliza o para solicitar que no se renueve automáticamente, $y$

VI. Los datos de la unidad especializada que la Institución de Seguros debe mantener en términos del art. 50 Bis de la Ley de Protección y Defensa al Usuario de Servicios Financieros.

El capítulo mencionado, a grandes rasgos determina la obligación de las aseguradoras de contener una cláusula en la cual se establezca:

1.- Forma en que se proporcionará la póliza (un plazo no mayor a 30 días). 
2.- El mecanismo de entrega de dicho documento.

3.- Medios alternativos al señalado en el número 2, para conseguir la póliza.

4.- Mecanismo de cancelación y/o solicitar no se renueve la póliza.

En relación con lo anterior, las instituciones de seguros y fianzas se encuentran obligadas a proporcionar los siguientes datos:

1.- Número de Póliza.

2.- Nombre comercial del producto contratado.

3.- Página de internet en donde se puedan consultar las condiciones generales de seguro.

4.- El contacto para la cancelación de la póliza y/o solicitar que no se renueve.

5.- Datos de la Unidad Especializada (UNE).

Para efectos de lo anterior, se desprende que la Circular Única de Seguros y Fianzas contiene una regulación que en un primer término facilita la contratación de los seguros vía electrónica, por la practicidad que la misma representa, sin que ello afecte a los derechos del asegurado de tener conocimiento de la documentación contractual a la que se hace referencia.

\section{MECANISMO DE PROTECCIÓN AL ASEGURADO}

\subsection{Criterios sobre sanas prácticas, transparencia y publicidad}

Por medio de las Disposiciones de Carácter General en Materia de Sanas Prácticas, Transparencia y Publicidad Aplicables a las Instituciones de Seguros emitidas por la CONDUSEF se establecen aquellas actividades que se apartan de las sanas prácticas y usos relativos al ofrecimiento y comercialización de las operaciones de seguros, así como la transparencia y publicidad de éstos.

Aquellas actividades que se alejan de las sanas prácticas son establecidas en el artículo tercero de esta disposición por medio de un catálogo o numerus clausus, el cual en un total de veintiún fracciones describe actividades como: proporcionar a los usuarios información engañosa o que induzca a error sobre los productos de los seguros que ofrecen o comercializan; ofrecer servicios de asistencia como si se tratara de productos de seguro; negar a los usuarios que lo soliciten, la documentación contractual en la que consten las características, derechos, obligaciones, limitaciones, coberturas o planes, deducibles, exclusiones, franquicias, riesgos, beneficios y costo de un producto para validar que coincida con lo ofertado o comercializado, previo a su contratación; abstenerse de proporcionar al usuario información de las principales características del producto de seguros, entre otras, las consecuencias de la rehabilitación, vigencia 
del producto, salvamentos y renovación automática; y negar al usuario la posibilidad de cancelar el contrato por la misma vía por la cual fue contratado, entre otras.

Además de las disposiciones establecidas en el artículo tercero, mencionado en el párrafo anterior, en el artículo cuarto de la misma disposición establece otras siete fracciones de actividades que se alejan de las sanas prácticas en casos de contratación por vía telefónica, las cuales describen actividades como: inducir al usuario a la contratación del seguro mediante engaños al utilizar términos ambiguos o que no concuerden con las características del producto; proporcionar información incompleta o que induzca al error sobre los productos de seguros ofrecidos; no proporcionar al usuario el número de póliza; y no establecer un medio cierto para la entrega de la documentación que avala la contratación del producto de seguro y que contiene las obligaciones y coberturas que ampara el mismo, entre otras.

Las disposiciones relativas a la transparencia establecen aquellas partes que forzosamente deben integrar un contrato de adhesión y otros documentos que deberán acompañarlos, como puede ser recibos de pago de primas, el folleto explicativo en el caso de productos de seguros de salud o anexos conteniendo los preceptos legales citados o indicando el lugar en que podrán consultarlos. Además de lo anterior, se establece que la solicitud de seguro que forme parte del contrato de adhesión, la carátula de la póliza y las condiciones generales deberán incluir cierta información como una leyenda referente a las limitaciones y exclusiones con las que cuenta el producto, número de registro en el Registro de Contratos de Adhesión de Seguros (" $\underline{\mathbf{R E C A S}}$ "), un anexo para consulta de abreviaturas de uso no común, datos de contacto de la CONDUSEF y datos de localización de la Unidad de Especialización de instituciones de seguros que atiende a quejas de los usuarios ("UNE").

En cuanto a la transparencia en la comercialización de seguros, se establece que los usuarios tendrán ciertas prerrogativas como el poder presentar una solicitud de aclaración o queja en el domicilio de la institución o en la UNE por cualquier medio con que se pueda comprobar fehacientemente su recepción. También podrán tanto el usuario solicitar la cancelación o terminación anticipada del contrato de seguro con excepción de los seguros que lo permiten, dando aviso a la institución de seguros y al usuario, respectivamente, cualquier tecnología o medio previsto en la LISF.

Las disposiciones emitidas por la CONDUSEF en cuanto al tema de la publicidad establecen que aquella que sea propuesta al usuario respecto de los productos ofrecidos no deberá ser engañosa, falsa u omisa; favorecer a la institución de seguros a expensas de un tercero; inducir a error o confusión, con respecto a productos propios o de terceros, ni incluir comparaciones falsas. También se establece que las instituciones de seguros deberán vigilar que las características del producto señaladas en la publicidad concuerden con las descritas en el contrato de adhesión y tendrán que señalar los requisitos que deben cumplir los usuarios para tener acceso a los beneficios ofertados.

En cuanto a los productos publicitados por medio de la página de internet de la institución, además de cumplir con lo establecido en la CUSF, las instituciones de seguros deberán proporcionar cierta información, como requisitos y modalidades para la contratación, los números telefónicos y correo electrónico de la UNE, datos de contacto de la CONDUSEF y explicación de las abreviaturas de uso no común que se utilicen. 


\title{
9.2 Protección de datos y privacidad
}

Puesto que los clientes tienen derecho a que sus transacciones financieras se realicen de manera confidencial, es obligación de las aseguradoras proteger la confidencialidad y seguridad de los datos personales proporcionados, frente a cualquier amenaza anticipada, riesgos para la seguridad o integridad de la información y contra el acceso no autorizado. Este derecho a la confidencialidad es protegido por diversos estatutos internacionales como las Directrices sobre Protección de la Privacidad y Flujos Transfronterizos de Datos Personales de la Organización para la Cooperación y el Desarrollo Económico (“'OCDE”) y las Directrices de la Organización de Naciones Unidas ("ㅇNU”) para la regulación de los archivos de datos personales informatizados. Es importante mencionar que el uso de la información médica y genética para la aceptación/ declinación y calificación de riesgos de vida es ahora un área principal de debate que no está al alcance de las buenas prácticas para el consumidor financiero reguladas por el Banco Mundial.

\subsection{Ventas atadas}

Con relación a las ventas atadas, el art. 18 de las Disposiciones de Carácter General en Materia de Sanas Prácticas, Transparencia y Publicidad establecen que

\begin{abstract}
“Tratándose de la oferta de productos financieros adicionales o ligados al producto solicitado por el Usuario, la Publicidad que lleve a cabo la institución de seguros deberá ser clara y precisa a fin de obtener el consentimiento del Usuario para su contratación, sin que medie engaño o se induzca al error, bajo la premisa de que es un derecho innegable del Usuario el contratar productos financieros a través de un tercero independiente".
\end{abstract}

\subsection{Alfabetización financiera}

Puesto que la educación, formación y capacitación financiera serían de gran ayuda para que los consumidores puedan presupuestar sus ingresos, ahorrar, invertir y protegerse contra riesgos y contra fraudes financieros, sería importante el desarrollo de programas de educación e información financiera para incrementar la alfabetización financiera de la población, los cuales deberían ser impartidos por una institución como el Banco de México en coordinación con otras instituciones gubernamentales y no gubernamentales. Esto es especialmente importante en la actualidad, ya que los productos y servicios financieros se vuelven cada vez más sofisticados y las familias deben asumir mayores responsabilidades financieras.

\section{CONCLUSIONES}

Una vez determinado que la regulación en México, ha seguido los principios de la Ley Modelo sobre el Comercio Electrónico (CNUDMI), la cual en términos generales contiene cuestiones básicas de la contratación mediante medios electrónicos (p. 9), se 
concluye tal como se expuso (pág. 3), que es muy difícil encontrar una completa armonía entre la norma jurídica y el constante cabio de la realidad en cuanto los medios electrónicos se trata, ya que atendiendo a la practicidad que dichos medios otorgan, legislar al ritmo de su cambio implica una cuestión materialmente imposible.

En virtud de lo anterior, lo prudente es determinar la facultad de celebración de contratos vía electrónica, sin que en ello medie limitación alguna, como lo es el soft law (p. 4), sin más que seguir los principios de la contratación electrónica (pp. 8 y 9), contendiendo en ellos como el más importante el de la "inalterabilidad del derecho preexistente", lo anterior para evitar contradicciones tal como se comentó (p. 3), y evidentemente siguiendo también la debida protección y seguridad jurídica de los usuarios de los servicios financieros (pp. 14, 15 y 16).

En ese orden de ideas, los tipos de contratación electrónica mencionados (pág. 6) entre otros, no son otra cosa que un facilitador (como lo es el uso de toda la tecnología), que supera la aplicación de la norma jurídica por el constante cambio, sin embargo, ello no implica que la naturaleza de los contratos, entre ellos el contrato de seguro, tenga que ser alterada.

Por su parte México, tal como se expuso (pp. 2, 9, 10, 11, 12, 13 y 14), sigue la ideología aquí expuesta y reconoce la contratación vía medios electrónicos, mientras que, en cuanto al contrato de seguro, determina únicamente obligaciones a la institución aseguradora para efectos de otorgar seguridad jurídica y protección para el usuario, sin que en ello medie limitación alguna.

\section{BIBLIOGRAFÍA}

1. Banco Mundial. (2012, junio). Buenas Prácticas para la Protección al Consumidor Financiero. Recuperado 25 febrero, 2020, de http://documentos.bancomundial.org/curated/ es/447021493843704521/pdf/70157-SPANISH-FinConsumerProtection-GoodPractices-SPANISH-FINAL.pdf

2. Medina Magallanes, Pablo. (septiembre 2018). En Búsqueda de la Real y Efectiva Protección del Consumidor de Seguros en México, en el Marco del Derecho Comparado. Trabajo de investigación para obtener el título de especialista en Derechos de Seguro dentro de los 43 Cursos de Especialización de Derecho de la Fundación General de la Universidad de Salamanca. Recuperado el 25 de febrero de 2020.

3. Isern SAlvat, María Rosa. (14 de febrero de 2014). Las Condiciones Generales del Contrato de Seguro y la Protección del Asegurado en el Derecho Español. Revista Boliviana de Derecho, 18, 98-121. Recuperado el 25 de febrero de 2020.

4. Gómez Santos, M. (2015, 5 noviembre). La Protección del Asegurado como Consumidor. Recuperado 25 febrero, 2020, de www.uclm.es/centro/cesco.

5. Arroyo Fernández, María Antonia; SAbido Rodríguez, Mercedes. (2010). Transparencia y Protección del Asegurado. Estudio sobre el sector asegurador en España 2010: los aspectos cualitativos de solvencia, II, 223-239. 25 de febrero de 2020, De Universidad de Extremadura Base de datos. 
6. Insights, Deloitte. (2018). El futuro de la regulación: principios para regular tecnologías emergentes. Recuperado 25 febrero, 2020, de https:/www2.deloitte.com/content/ dam/Deloitte/pe/Documents/risk/Futuro\%20de\%201a\%20regulaci\%C3\%B3nRevII\%20(002).pdf. 